\title{
New MR sequences in daily practice: susceptibility weighted imaging. A pictorial essay
}

\author{
Roberto Gasparotti • Lorenzo Pinelli • Roberto Liserre
}

Received: 13 August 2010/Revised: 22 November 2010 /Accepted: 15 February 2011 /Published online: 26 March 2011

(C) European Society of Radiology 2011

\begin{abstract}
Background Susceptibility-weighted imaging (SWI) is a relatively new magnetic resonance (MR) technique that exploits the magnetic susceptibility differences of various tissues, such as blood, iron and calcification, as a new source of contrast enhancement. This pictorial review is aimed at illustrating and discussing its main clinical applications.

Methods SWI is based on high-resolution, threedimensional (3D), fully velocity-compensated gradientecho sequences using both magnitude and phase images. A phase mask obtained from the MR phase images is multiplied with magnitude images in order to increase the visualisation of the smaller veins and other sources of susceptibility effects, which are displayed at best after postprocessing of the $3 \mathrm{D}$ dataset with the minimal intensity projection (minIP) algorithm.

Results SWI is very useful in detecting cerebral microbleeds in ageing and occult low-flow vascular malformations, in characterising brain tumours and degenerative
\end{abstract}

R. Gasparotti $\cdot$ L. Pinelli $\cdot$ R. Liserre

Neuroradiology Unit, Department of Diagnostic Imaging,

University of Brescia,

Spedali Civili,

Brescia, Italy

L. Pinelli

e-mail: lorepine@libero.it

R. Liserre

e-mail: rliserre@libero.it

R. Gasparotti ( $\square)$

U. O. Neuroradiologia, Dipartimento di Diagnostica per

Immagini, Università degli Studi di Brescia,

Spedali Civili di Brescia, Piazza Spedali Civili 1,

25123, Brescia, Italy

e-mail: gasparo@med.unibs.it diseases of the brain, and in recognizing calcifications in various pathological conditions. The phase images are especially useful in differentiating between paramagnetic susceptibility effects of blood and diamagnetic effects of calcium. SWI can also be used to evaluate changes in iron content in different neurodegenerative disorders.

Conclusion SWI is useful in differentiating and characterising diverse brain disorders.

Keywords Brain · Magnetic resonance imaging . Susceptibility weighted imaging

\section{Introduction}

Susceptibility weighted imaging (SWI) is a relatively new magnetic resonance (MR) technique that provides innovative sources of contrast enhancement visualising the changes in magnetic susceptibility that are caused by different substances like iron, haemorrhage or calcium. The basic concept of this technique is maintaining phase information into the final image, discarding phase artefacts and keeping just the local phase of interest.

Sensitivity to susceptibility effects increases, progressing from fast spin-echo (SE) to conventional SE to gradientecho (GE) sequences, from $\mathrm{T} 2$-weighting to $\mathrm{T} 2 *$-weighting, from short to long echo times and from lower to higher field strengths. Before the clinical implementation of SWI, susceptibility imaging relied only on GE sequences. SWI differs significantly from a $\mathrm{T} 2 *$-weighted GE sequence: it is based on a long echo time (TE) high-resolution, flowcompensated, three-dimensional (3D) GE imaging technique with filtered phase information in each voxel. The combination of magnitude and phase data produces an enhanced contrast magnitude image that is particularly 

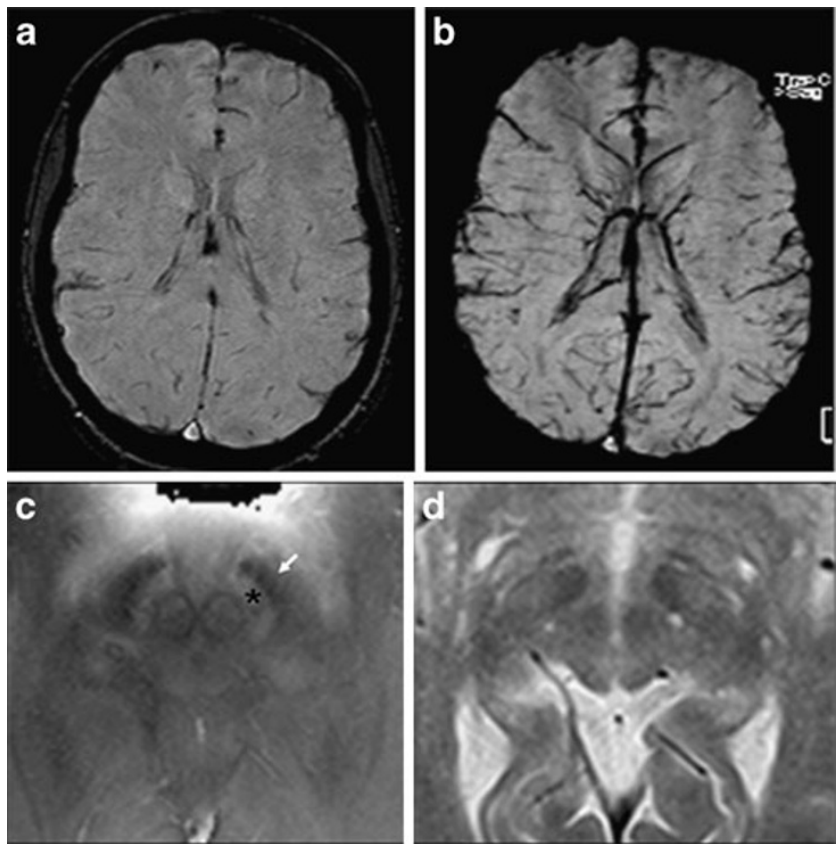

Fig. 1 Normal subject. a SWI magnitude image. b SWI, minimal intensity projection (minIP; $10 \mathrm{~mm}$ ) image: cortical and subependymal veins are well displayed. c SWI, phase map: the distinction between the pars reticulata (arrow) and compacta (asterisk) of the substantia nigra is enhanced compared with conventional fast SE and GE sequences. The outer margins of the red nucleus are also better displayed (see $\mathbf{d}$ for comparison). $\mathbf{d}$ T2-weighted axial image sensitive to haemorrhage, calcium, iron storage and slow venous blood, thus allowing a significant improvement compared with T2* GE sequences. After imaging acquisition, incidental phase variations due to static magnetic field heterogeneities are removed. The phase mask is then multiplied with the magnitude data to enhance the visualisation of vessels or foci with susceptibility effects [1]. SWI is therefore especially helpful in the detection of calcifications and microhaemorrhages, which are both characterised by low signal. The evaluation of the corrected phase images allows the differentiation between the two substances, as calcifications appear bright because of a positive phase shift and haemorrhages appear dark because of a negative phase shift.

A supplementary source of information in SWI is primarily associated with the magnetic susceptibility differences between oxygenated and deoxygenated haemoglobin. SWI represents a technical improvement in "high-resolution blood oxygenation level-dependent venography” (HRBV), originally developed by Reichenbach et al. [2], which was based on 3D long TE, flow-independent GE sequences and manipulation of the images with the phase data. The paramagnetic properties of deoxyhaemoglobin [BOLD (blood oxygen level dependent) effect] and the prolonged T2* of venous blood were used as an intrinsic contrast agent, leading to a phase difference between vessels containing deoxygenated blood and surrounding brain
Fig. 2 Recent onset of altered mental status and cognitive impairment in a 67-year-old man. a Fluid attenuated inversion recovery (FLAIR) MRI: diffuse hyperintensity of the white matter, more pronounced in the right parietal region. b Diffusion-weighted imaging (DWI), apparent diffusion coefficient (ADC) map: the whitematter hyperintensity represents vasogenic oedema. c GE T2* weighted image: small punctate hypointense foci in the right parietal cortex (open arrow). d SWI minIP: increased visualisation of markedly hypointense foci surrounding the white matter abnormalities which correspond to multiple cerebral microbleeds. On the basis of SWI imaging findings, CAA with diffuse inflammatory changes was suspected and steroid therapy was administered. e Follow-up MRI at 3 months showed significant reduction of the vasogenic oedema
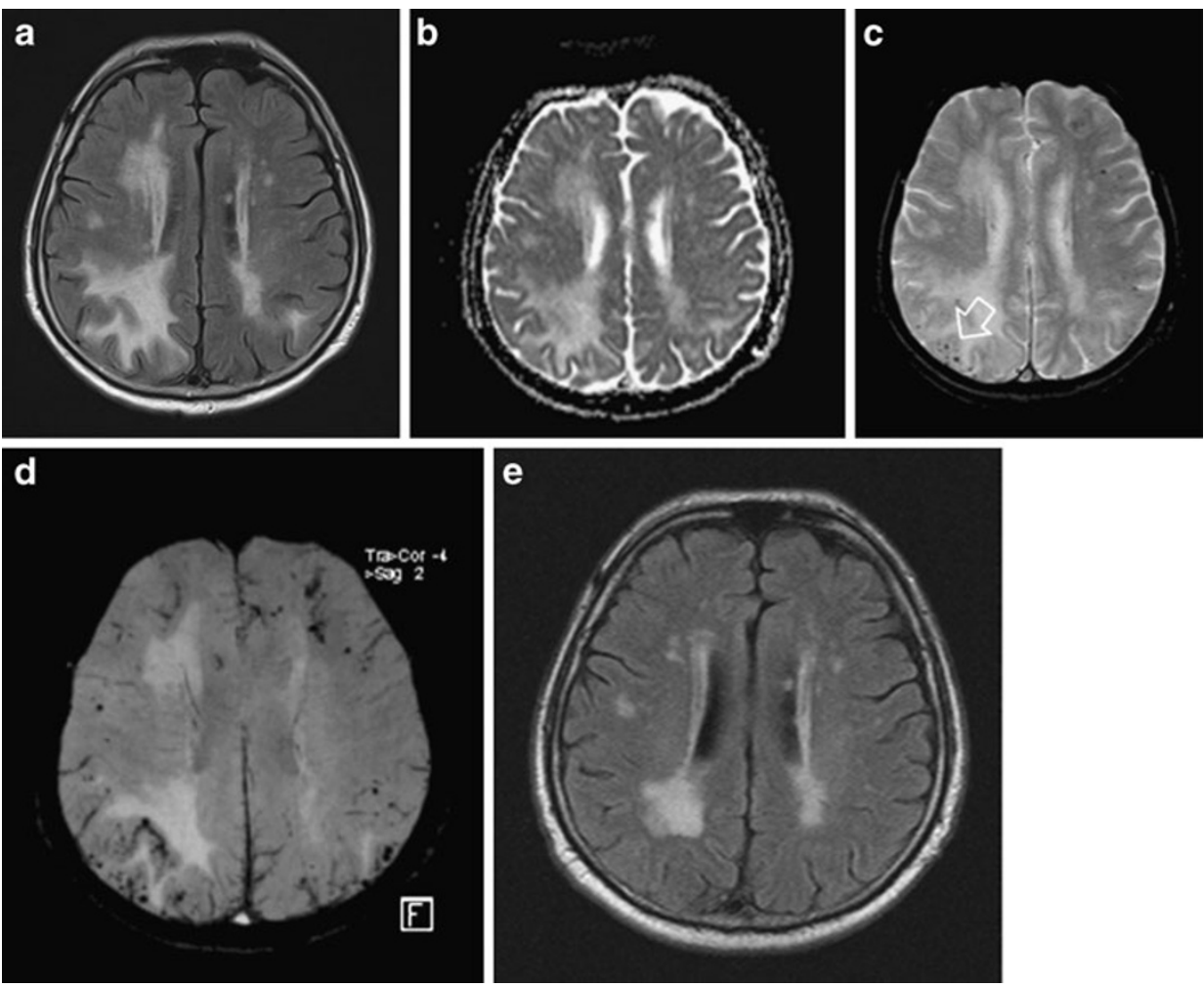

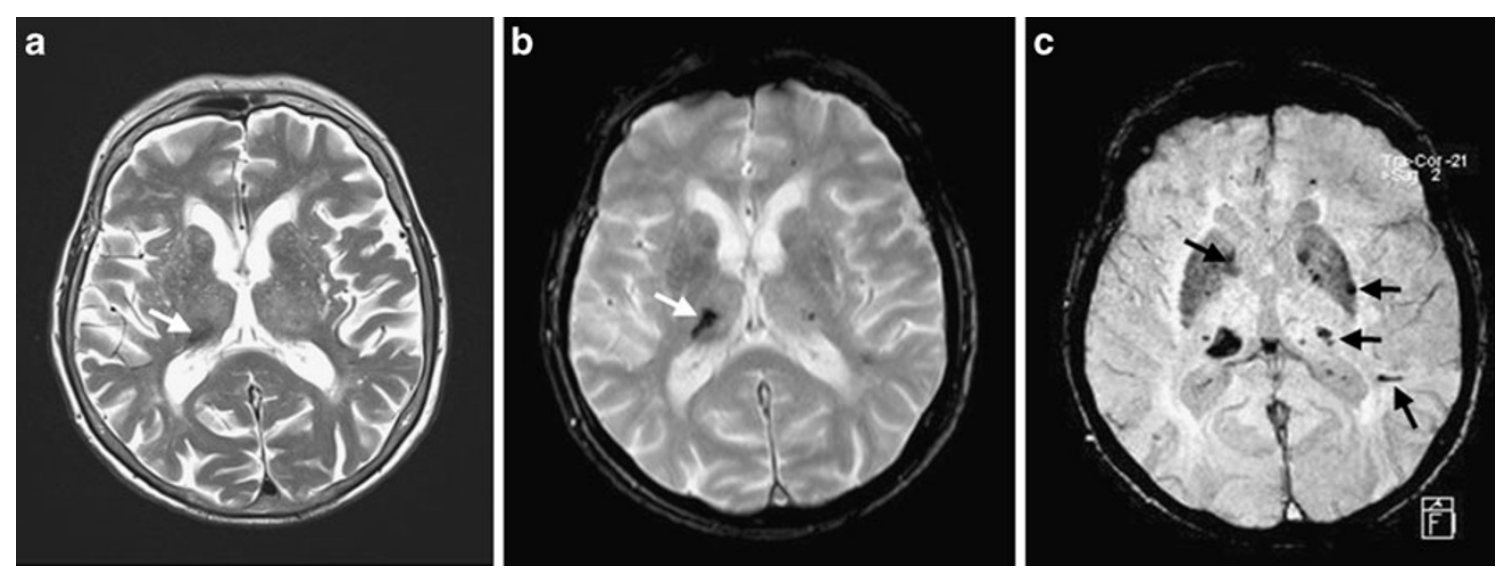

Fig. 3 Patient with long-standing hypertension. a T2-weighted image shows a thin hypointense band in the right thalamus, which represents haemosiderin deposition (arrow). b GE T2*-weighted image shows multiple microbleeds, the largest one in the right thalamus (arrow). c

tissue, resulting in signal intensity cancellation. Thus, deoxyhaemoglobin can behave like a contrast agent with long TEs for differentiating arteries from small veins, which can be as small as $100-200 \mu \mathrm{m}$ and therefore difficult to detect with conventional MR angiography techniques, such as time of flight (TOF) or phase contrast (PC) [3]. For this reason, the phase-added information that is usually not available in the conventional magnitude image makes SWI well suited for the visualisation of very small vessels such as the caput medusae of venous angiomas and telangiectasias as a result of a combination of slow flow with changes in deoxyhaemoglobin concentration [4].

Latest advances have allowed the technique to be refined, thereby expanding its clinical applicability to brain imaging as a complementary source of information to conventional $\mathrm{T} 1$-weighted and $\mathrm{T} 2$-weighted imaging sequences.

This pictorial essay is aimed at showing the most relevant clinical applications of SWI.
SWI minIP: the number of identifiable microbleeds is increased compared with GE images. They can be recognised bilaterally in the basal ganglia and in the subcortical temporal white matter (arrows)

\section{Technical aspects}

At our institution, MR imaging (MRI) is performed by using a 1.5-T system (Magnetom Avanto; Siemens, Erlangen, Germany) with a 12-channel head coil. SWI is obtained with a long-TE, fully flow-compensated 3D GE sequence with the following parameters: repetition time (TR)/TE, 49/40 ms; flip angle, $15^{\circ}$; rectangular field of view (FOV), 7/8; matrix size, $280 \times 320$; slice thickness, $1.6 \mathrm{~mm}$ (80 slices in a single slab matrix size); iPAT factor, 2; acquisition time, $5 \mathrm{~min}$. Images are acquired in the axial plane parallel to the bicommissural line.

The SWI sequences are reconstructed with the minimum intensity projection algorithm (minIP) and multiplanar reformation (MPR) techniques to obtain images with thickness $(3-10 \mathrm{~mm})$ and position comparable to those of conventional sequences. The minIP algorithm has the characteristic to enhance the visualisation of veins while attenuating the signal from the brain tissue (Fig. 1).
Fig. 4 Alzheimer's disease. a Coronal reformatted

T1-weighted MP-RAGE section depicts marked atrophy of the hippocampi. b Coronal reformatted SWI section shows a cerebral microbleed in the right frontal white matter (arrow)
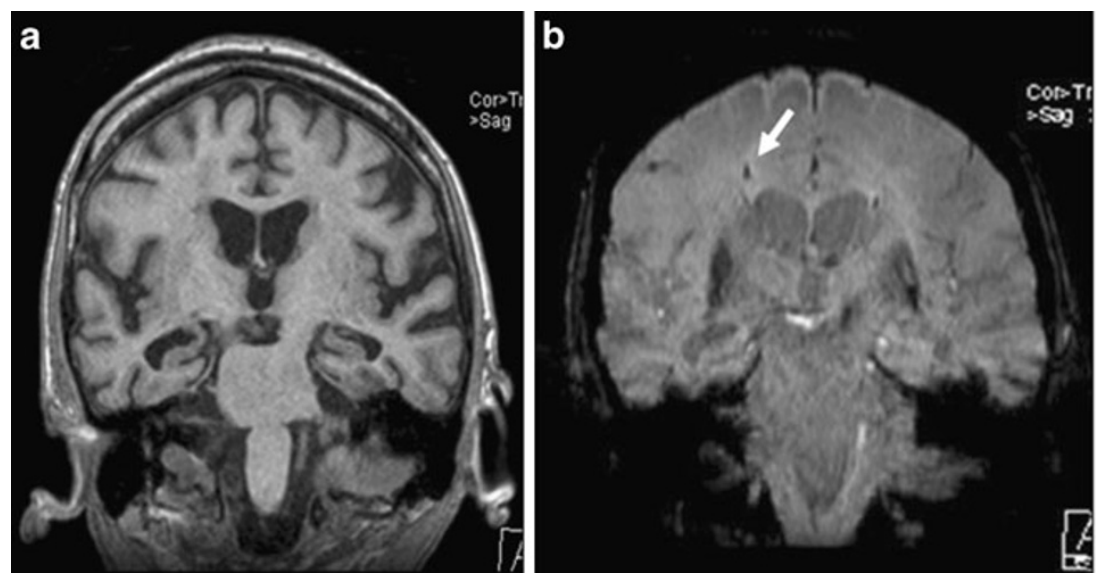
SWI sequences have some intrinsic disadvantages, which are mainly represented by artefacts caused by undesirable sources of magnetic susceptibility that occur at air-tissue interfaces, therefore limiting the investigation of areas next to paranasal sinuses and temporal bone. The "blooming artefact", useful in most cases, might also not be needed in some situations, producing normal tissue signal cancellation and loss of anatomical borders. The sequence acquisition time on a 1.5 -T system ranges from 5 to $8 \mathrm{~min}$, depending on the spatial resolution and the coverage of the brain needed, leading to an increased incidence of movement artefacts.

Imaging at a high field strength has some advantages over $1.5 \mathrm{~T}$ in the delineation of even smaller vessels belonging to the venous network, with shorter imaging times because of the higher signal-to-noise ratio, higher spatial resolution and increased susceptibility effects [5]. However susceptibility-based signal loss and severe image distortion caused by air-tissue interfaces or other sources of local field heterogeneity are much more severe at higher
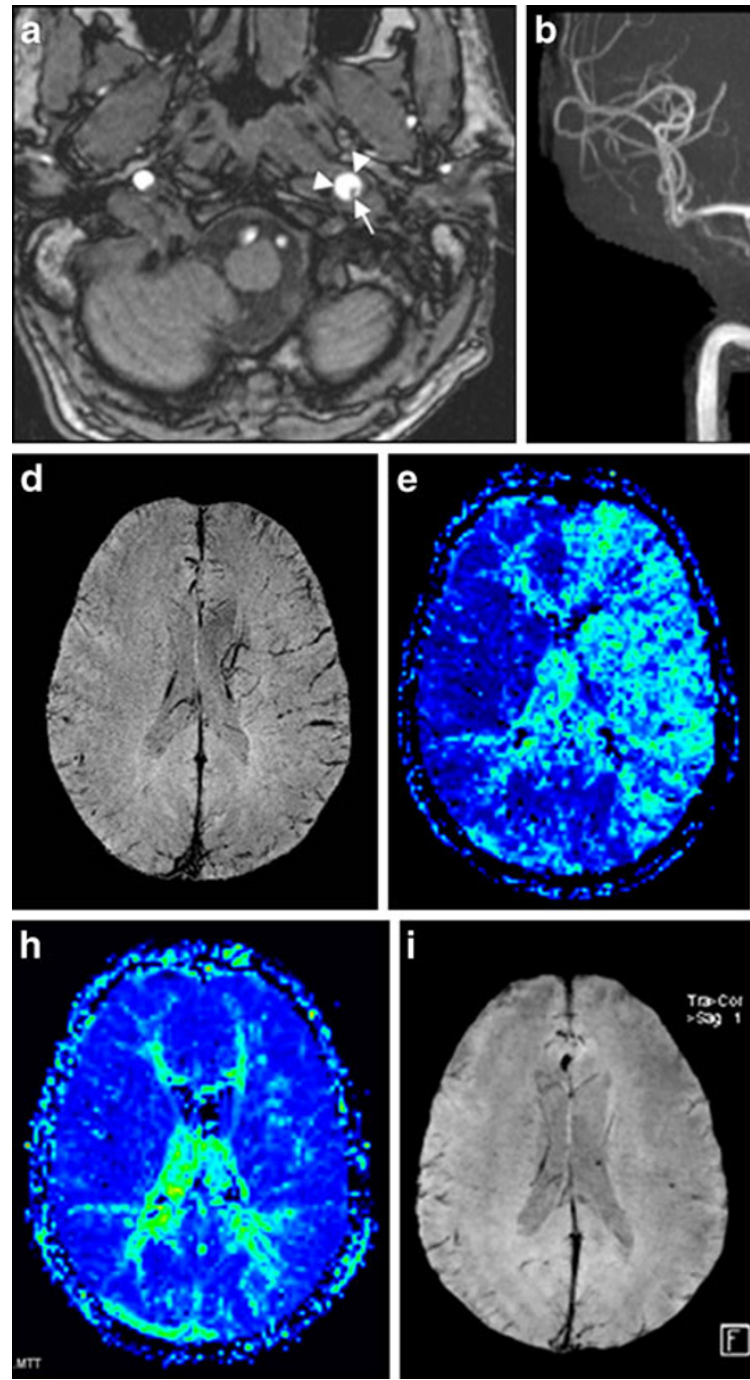

Fig. 5 Left internal carotid artery (ICA) dissection with acute watershed infarct in a 42-year-old man with sudden onset of speech disturbance. a MR angiography, single partition at the level of the skull base showing dissection of the left ICA, which is characteristically enlarged with highsignal-intensity methaemoglobin (arrowheads) representing the intramural thrombus and a small eccentric lumen (arrow). b MR angiography, coronal MIP: narrowing of the ICA in its vertical segment just below the skull base (arrow). c DWI, axial image: acute watershed infarct in the left centrum semiovale. d SWI minIP: improved visualisation of the veins of the left cerebral hemisphere, which is related to increased oxygen extraction in the ischaemic penumbra and corresponds to the hypoperfusion deficit shown by the mean transit time (MTT) map. e Perfusion MRI, MTT map, showing delayed transit time in the left middle cerebral artery (MCA) territory. $\mathbf{f}$ Intra-arterial digital subtraction angiography (DSA), left carotid angiogram, confirming the ICA stenosis (arrow). $\mathbf{g}$ Intra-arterial DSA: the patient was treated using stent placement, which resulted in partial restoration of the vessel lumen. h Perfusion MRI, MTT map $24 \mathrm{~h}$ after stent placement: complete resolution of the perfusion deficit. i SWI minIP, normalisation of the venous drainage of the left hemisphere 
fields, thus reducing SWI's usefulness in the evaluation of the posterior fossa and skull base.

SWI has been investigated at $3 \mathrm{~T}$ with excellent results, especially concerning brain tumours and detection of cerebral microbleeds $[6,7]$.

\section{Clinical applications}

Cerebral amyloid angiopathy

Sporadic cerebral amyloid angiopathy (CAA) is a common small-vessel disease associated with ageing, dementia and Alzheimer's disease, that can only be diagnosed histopathologically following biopsy or at post-mortem examination. It consists of deposition of amyloid protein within the small and medium-sized cerebral arteries, which is likely responsible for increased vessel fragility and it is one of the major causes of lobar intraparenchymal haemorrhages in the elderly [8].

Computed tomography (CT) and conventional MR techniques are usually unable to show cerebral microbleeds (CMBs), which can be frequently observed on T2*-weighted gradient-echo MRI and have a typical lobar distribution [9]. Recent findings indicate that CMBs in the general elderly population are relatively common and are even more frequently observed in patients with $\mathrm{AD}[10]$.

SWI is much more sensitive than GE sequences in demonstrating CMBs in and around the arteriole vessel wall, which appear as black dots better displayed by minIP images (Fig. 2). Histologically, they represent perivascular clusters of haemosiderin-laden macrophages resulting from leakage from cerebral small vessels [11]. Higher field strengths, as expected, can improve CMB detectability, increasing the lesion's contrast enhancement [7].

CAA is also characterised by white matter hyperintensities on conventional MRI sequences, which have been associated with cognitive impairment [12]. Vascular amyloid deposition is believed to be involved in the pathophysiological mechanisms that determine white matter hypoperfusion through either vessel stenosis or vascular dysfunction [13].

CAA should also be suspected in elderly patients with clinical signs of a progressive encephalopathy syndrome with seizures, in which extensive white matter abnormalities are discovered in brain MRI together with multiple CMBs with lobar and subcortical distribution (Fig. 2). It has been recently reported that this combination of MR findings should be interpreted as CAA-related inflammation, which can be treated with steroid therapy with a prompt resolution of the symptoms [14]. In those cases, the demonstration of an APoE $\varepsilon 4 \varepsilon 4$ genotype can definitely support the neuroradiological diagnosis. Hypertensive encephalopathy and especially posterior reversible encephalopathy syndrome (PRES), when the signal abnormalities of the white matter are more symmetrical and located in parieto-occipital regions, should be taken into consideration for differential diagnosis.

\section{Hypertensive encephalopathy}

Hypertensive encephalopathy is characterised by multiple CMBs which are usually silent and can be discovered when the patient is investigated with MRI in order to understand the cause of an intraparenchymal haemorrhage located outside the basal ganglia. SWI is more sensitive to CMBs than $\mathrm{T} 2 *$-weighted GE sequences in blood pressure (BP)related small vessels disease. CMBs are usually discovered both in deep basal ganglia and subcortical white matter (Fig. 3). Histologically they represent focal accumulations of haemosiderin-containing macrophages in the perivascular space of small brain vessels, indicating previous extravasation of blood, and are often associated with the presence of a symptomatic haemorrhage in the corresponding area. The number of CMBs, which remain detectable for years, is significantly associated with BP levels. Moreover, the presence of deep CMBs can be a
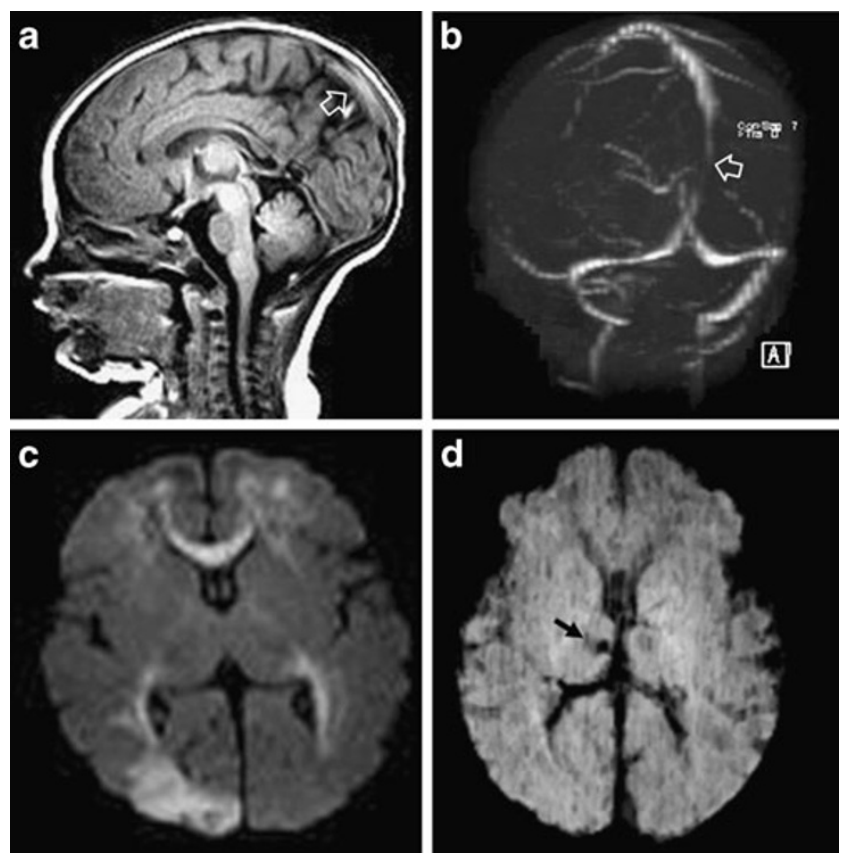

Fig. 6 Epileptic seizures and sagittal sinus thrombosis in a 3-year-old child. a Sagittal T1-weighted image demonstrates hyperintensity of the posterior third of the sagittal sinus (open arrow). b MR venography confirms the thrombosis of the posterior third of the sagittal sinus ending at the torcular (open arrow). c DWI, $b=1,000$ image demonstrates a right occipital acute ischaemic lesion. d SWI minIP shows a right thalamic haemorrhagic lesion in the vascular territory of the right deep internal cerebral vein (arrow) 

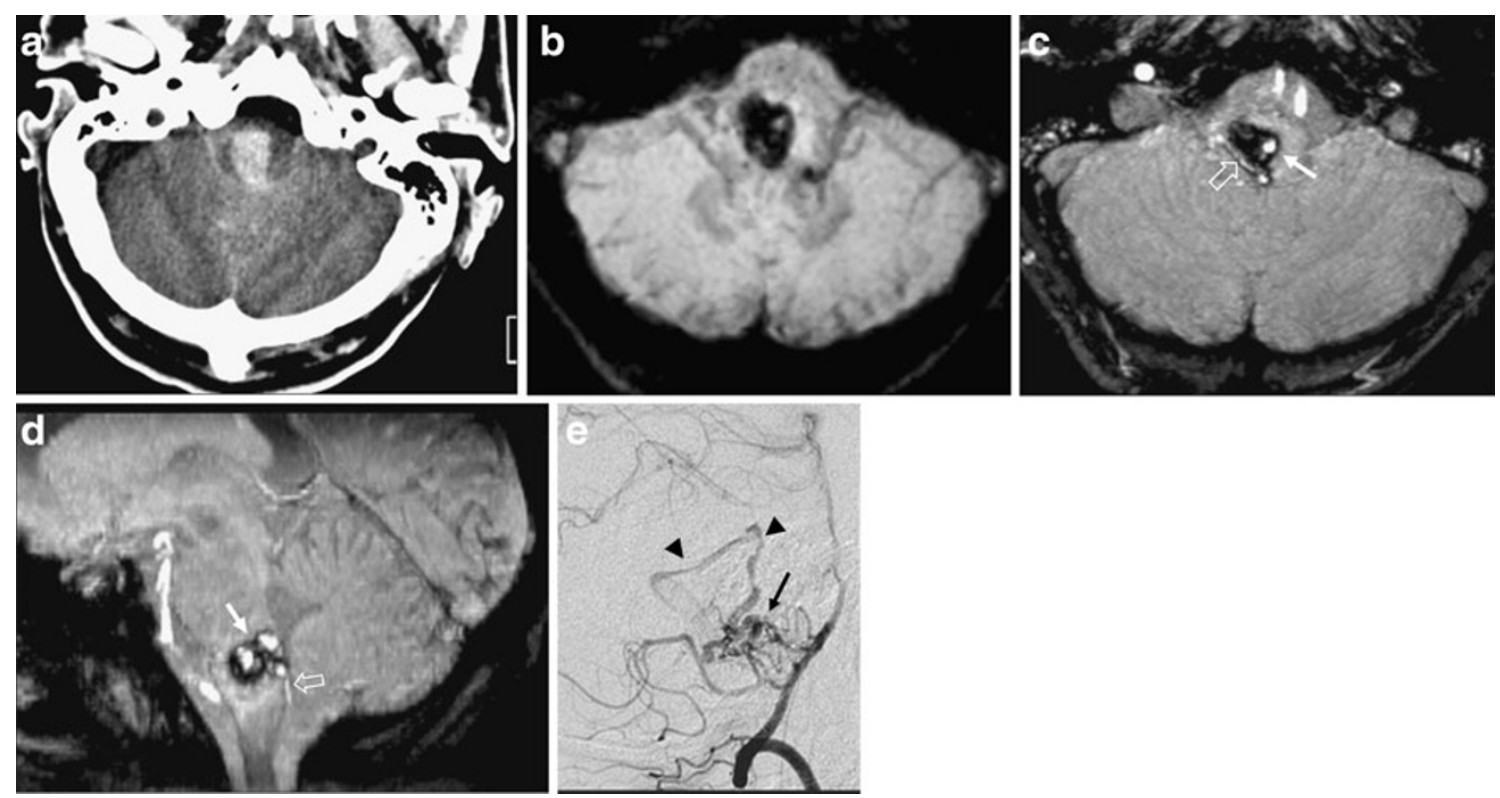

Fig. 7 Brainstem haemorrhage in a 43-year-old patient. a CT shows an acute haemorrhagic lesion located in the medulla oblongata. $\mathbf{b}$ The SWI minIP image clearly depicts the brainstem haemorrhage, which is markedly hypointense. c, d SWI, axial and reformatted sagittal images, processed with a maximum intensity projection algorithm (MIP), show flow-related enhancement within the haemorrhagic lesion (arrow) and a hypertrophied right posterior inferior cerebellar artery (PICA; open arrow) which are consistent with a small ruptured brainstem AVM. e Cerebral angiography, selective injection of the right vertebral artery: brainstem AVM (arrow) fed by branches of the right PICA and early venous drainage into a cerebellar vein (arrowheads)
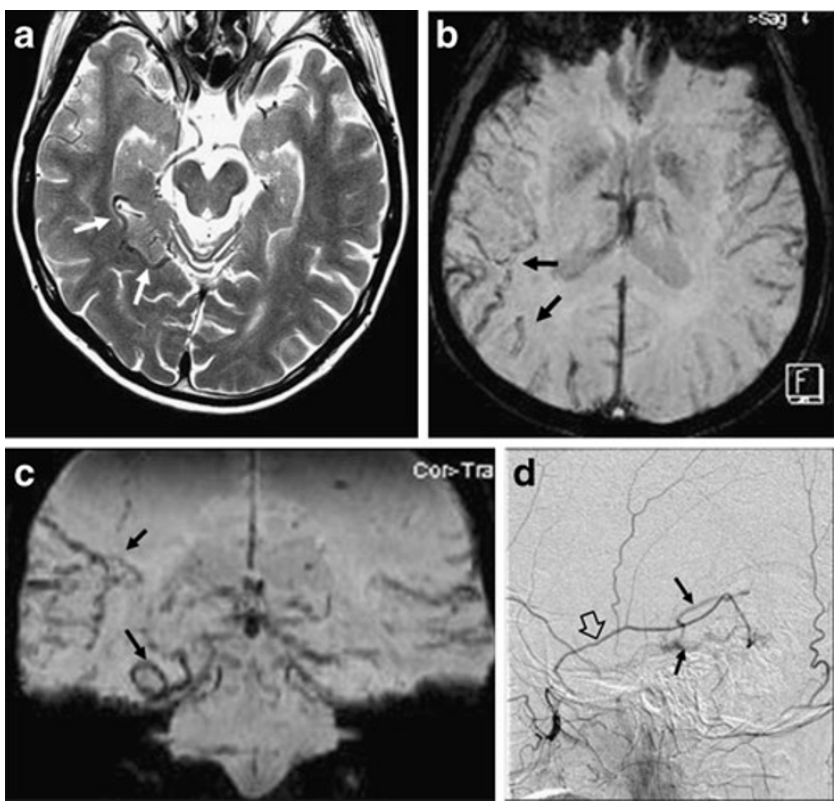

Fig. 8 Patient with headache and pulsatile tinnitus of the right ear. a T2-weighted image shows a dilated right temporal vein (arrows). b, c SWI axial and coronal reformatted minIP images show dilated right temporal veins (arrows), which arouse suspicion of a tentorial dural AV fistula. d Cerebral angiography, selective injection of the right external carotid artery: dural AV fistula of the right tentorium, fed by the right middle meningeal artery (open arrow), with early venous drainage into the tentorial and temporal veins (arrows) useful marker of BP-related small vessels disease, helping in the differential diagnosis with CAA[15].

\section{Neurodegenerative diseases}

Iron deposition increases in the brain as a function of age, primarily in the form of ferritin and particularly in oligodendrocytes, but also in neurons and microglia. Typical sites of iron deposition include the globus pallidum, substantia nigra, and red and dentate nuclei. Ferritin is paramagnetic and produces strong susceptibility effects on T2*-weighted images. SWI filtered-phase images are particularly suitable for showing increased iron content in the brain ([16]. Abnormally elevated iron levels are evident in many neurodegenerative disorders, including Parkinson's disease, Alzheimer's disease, Huntington's disease and amyotrophic lateral sclerosis.

The ability to measure the amount of ferritin in the brain can be used for a better understanding of the progression of the disease and is also helpful in predicting the treatment outcome.

Phase images allow a better distinction between the pars compacta and the pars reticulata of the substantia nigra, which contains iron (Fig. 1). As in patients with idiopathic Parkinson's disease, there is evidence of increased iron in the substantia nigra [17], SWI sequences can be proposed 

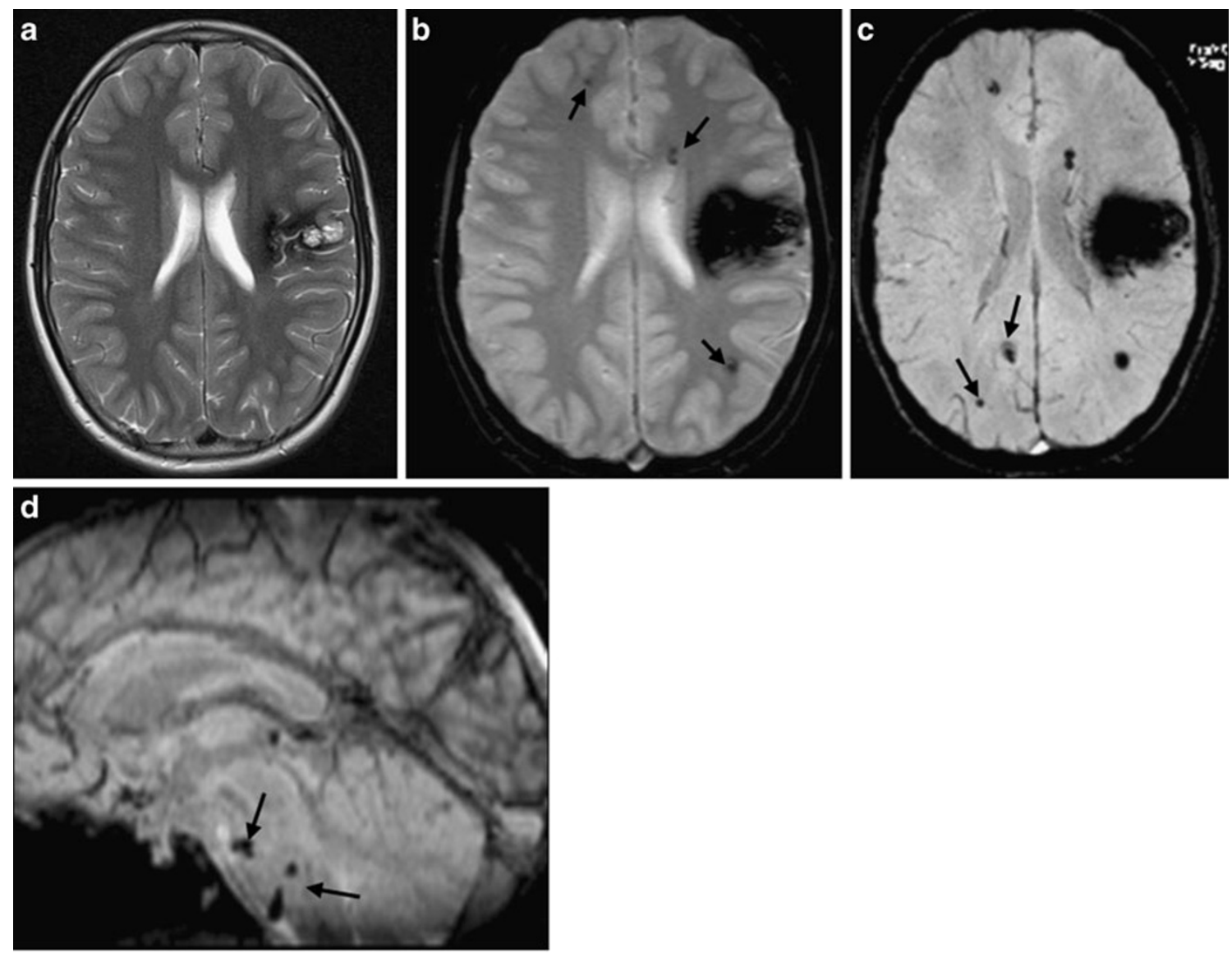

Fig. 9 Multiple cavernous malformations in a 32-year-old man investigated for intraparenchymal haemorrhage. The patient turned out to be affected by familial cavernous angiomatosis. a T2-weighted image demonstrates a left frontal cavernous malformation surrounded by a thick ring of haemosiderin. b GE T2*-weighted image shows multiple punctate hypointense foci located in both hemispheres, corresponding to small cavernous malformations (arrows). c SWI minIP: there are supplemental cavernous malformations that are not detected by GE sequences (arrows). d SWI minIP, reformatted sagittal section: multiple small cavernous malformations located in the brainstem (arrows)
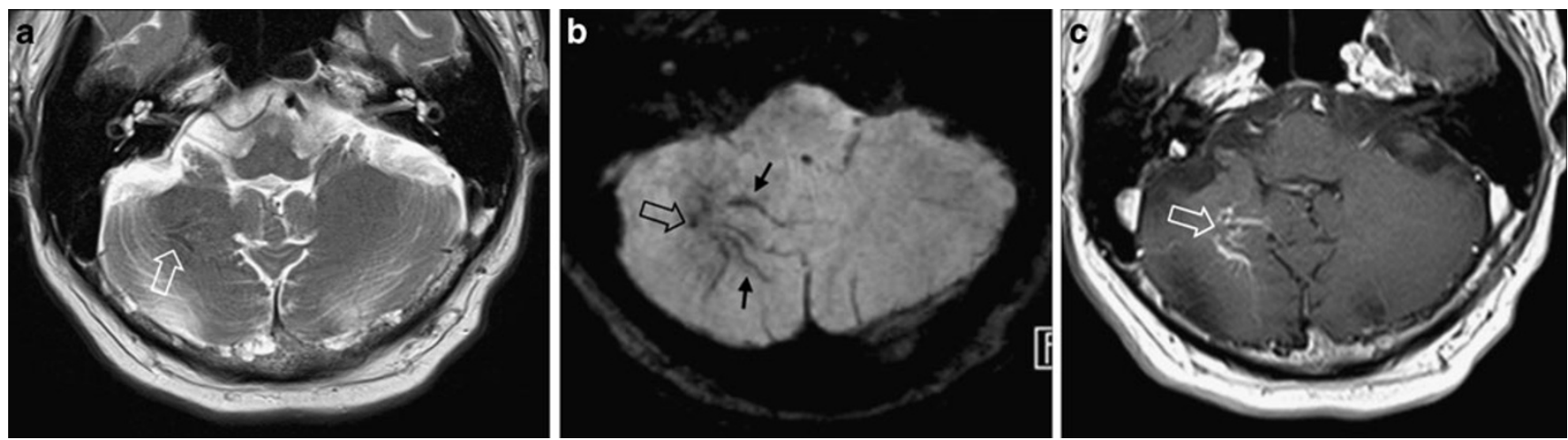

Fig. 10 Incidental discovery of a cerebellar developmental venous anomaly in a 48-year-old man investigated for right-sided sensorineural hearing loss. a T2-weighted image shows thin hypointense bands of flow void, in the right cerebellar hemisphere (open arrow). b SWI minIP demonstrates multiple thin medullary veins (arrows) that converge into a dilated collector vein (open arrow), consistent with a cerebellar venous angioma, better defined as developmental venous anomaly. c Post-contrast T1-weighted axial image shows the enhancing dilated veins (open arrow) 

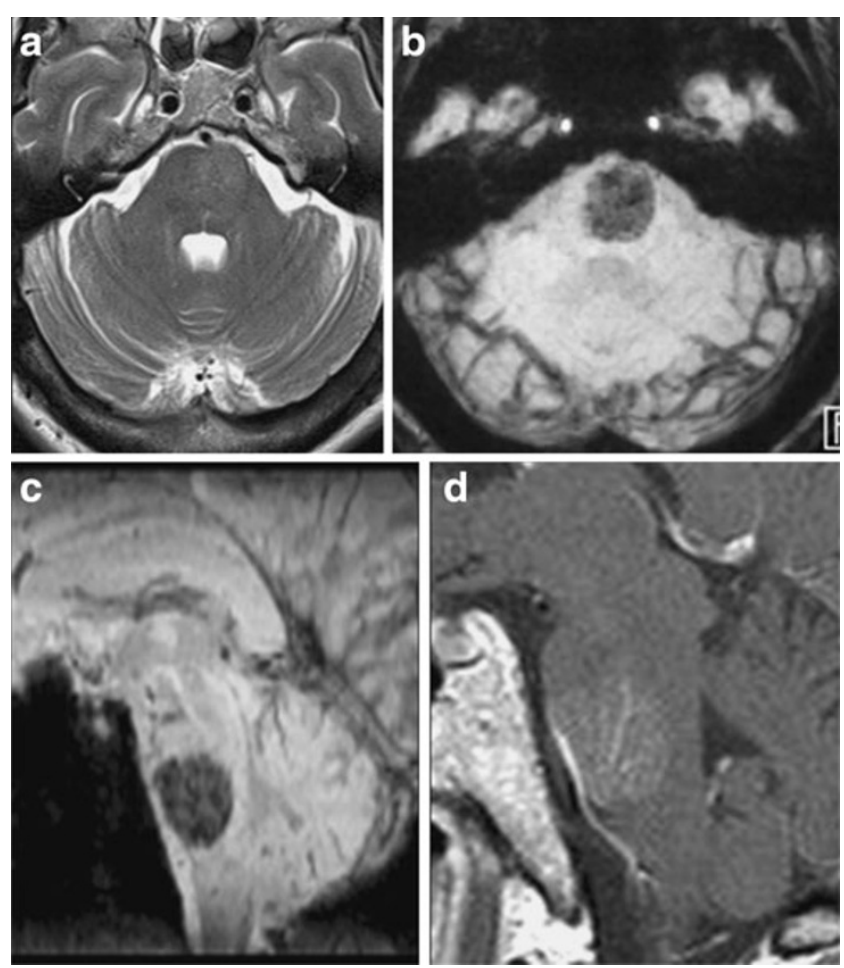

Fig. 11 Incidental discovery of a pontine telangiectasia in a 34-yearold woman investigated for migraine. a T2-weighted image barely shows the abnormality. SWI minIP axial (b) and sagittal reformatted section (c) show a markedly hypointense lesion with regular margins, located in the pons. d Post-contrast sagittal T1-weighted image. The capillary telangiectasia is characterised by mild enhancement with an arbor-like pattern

as a useful imaging tool to identify iron deposition as a biomarker for disease progression, although longitudinal studies are required to support the usefulness of this specific application [18]. Accurate localisation of the subthalamic nucleus can also be achieved in the SWI phase maps at $3 \mathrm{~T}$, allowing safe direct targeting for placement of electrodes in the treatment of Parkinson's disease [19].

In $70-98 \%$ of patients with Alzheimer's disease, intravascular amyloid deposition is found at autopsy [20] and CMBs are commonly observed. Recent evidence has shown that patients with multiple CMBs have more white matter hyperintensities and perform worse on mini mental state examination compared with patients with no CMBs, despite similar disease duration [21]. A significant correlation has also been reported recently between patients with at least one hypointensity in GRE-T2* imaging and those homozygous for the apolipoprotein E $\varepsilon 4 \varepsilon 4$ gene, a wellknown risk factor for Alzheimer's disease (Fig 4) [22].

Stroke

SWI has been demonstrated to be very useful in the acute phase of stroke for several reasons. First of all, it is very sensitive to the presence of $\mathrm{CMBs}$, whose early identification is believed to predict the probability of potential haemorrhagic transformation after thrombolytic treatment [23]. It has also been hypothesised that CMBs can represent a link between cerebral haemorrhage and ischaemia [24].

SWI is also capable of identifying the acute intravascular clot in the main and distal branches of the cerebral arteries [25].

The evaluation of the intravascular content of deoxyhaemoglobin in hypoperfused brain is one of the most interesting applications of SWI. This technique has been proved to be useful for the assessment of tissue viability in patients with hyperacute cerebral ischaemia, as it provides functional information about the ischaemic penumbra. In acute arterial stroke, flow reduction or absence causes an
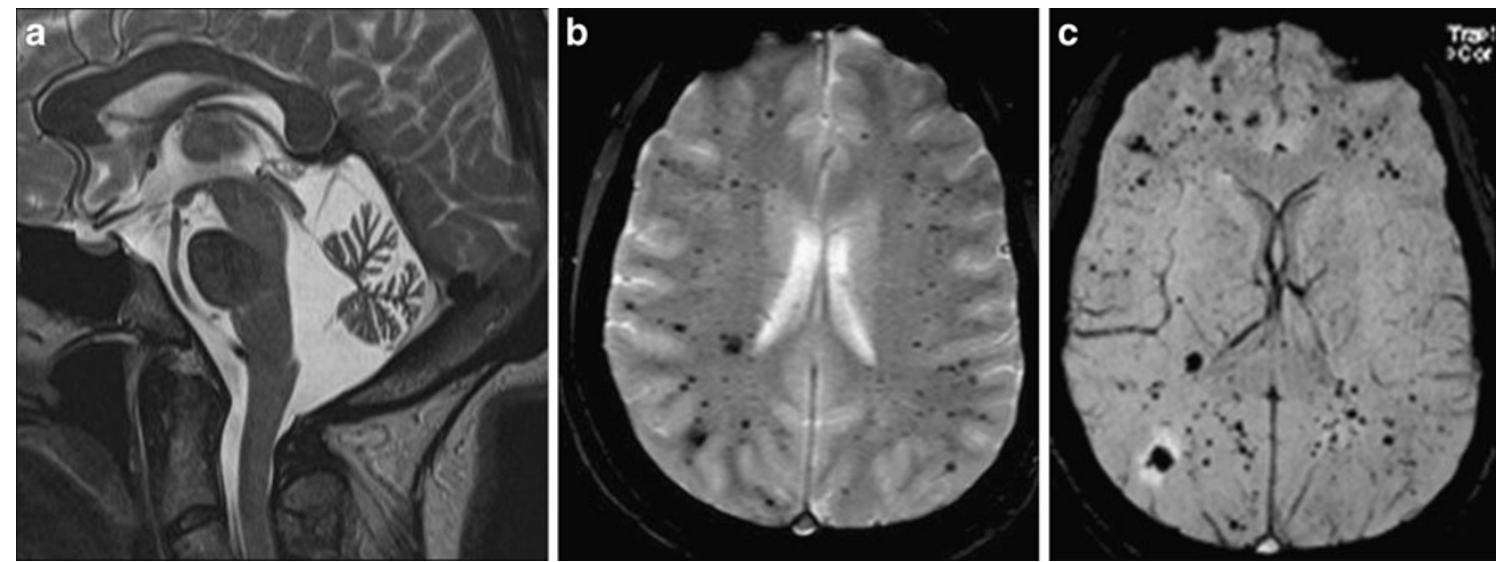

Fig. 12 Ataxia telangiectasia. A 31-year-old woman with a diagnosis of ataxia telangiectasia by the age of 7 , investigated for dizziness and headache. a Sagittal T2-weighted image demonstrates severe atrophy of the cerebellar vermis. b GE T2*-weighted image reveals multiple hypointense foci located in both hemispheres. c SWI minIP: additional hypointense black spots can be identified, corresponding to haemosiderin deposits presumably due to gliovascular nodules with perivascular haemorrhage 
increase in oxygen extraction, leading to a growth in the amount of deoxyhaemoglobin in the hypoperfused brain tissue. Deoxyhaemoglobin represents a supplementary source of contrast enhancement in SWI because of its paramagnetic properties, which determine a reduction in T2* as well as a phase difference between the vessel and its surrounding parenchyma. It appears markedly hypointense on both magnitude image and phase images. As the T1 and $\mathrm{T} 2$ properties of blood depend on the oxygen saturation of the blood, there are differences between arterial and venous vessels that cannot be discovered with conventional sequences. The visualisation of draining veins within areas of impaired perfusion allows the identification of penumbral brain tissue in a different way from the current perfusion-weighted imaging (PWI) techniques (Fig. 5) [26].

Cerebral venous sinus thrombosis

SWI has become a functional method for evaluating cerebral venous sinus thrombosis (CVST) by demonstrating engorgement of the venous system as a result of venous hypertension and collateral slow flow. Dural sinus thrombosis causes an increase in deoxyhaemoglobin concentration in the veins involved that appears as a prominent area of hypointense signal intensity on SWI; if CVST is treated successfully, this effect will disappear [26]. SWI is also capable of identifying the thrombosed pial vein, which is responsible for the development of oedema into the white matter, due to its high sensitivity in the demonstration of an intravascular clot. SWI can depict at the same time the features of parenchymal or extra-axial haemorrhages that can occur in the case of infarction, with higher sensitivity compared with conventional GE sequences (Fig. 6).

\section{Cerebral vascular malformations}

Cerebral arteriovenous malformations (AVMs) are easily displayed by conventional MRI and MR angiography because of their characteristic high flow, whereas venous malformations such as cavernomas, developmental venous anomalies and capillary telangiectasias cannot be adequately visualised without contrast medium administration and GE sequences, as they mainly consist of slow-flow small vessels. MR angiography techniques are often inadequate for visualising small vessels with slow flow. On the contrary, SWI sequences are well suited for the visualisation of very small vessels, such as the caput medusae of venous angiomas and telangiectasias as a result of a combination of slow flow with changes in deoxyhaemoglobin concentration [5]. The combined information derived from both phase and magnitude images is responsible for enhanced visualisation of such lesions.
Fig. 13 Diffuse axonal injury in a 14-year-old boy who had severe TBI following a motorcycle crash. Brain MR study was performed three days after the trauma, when the patient was in a severely comatose state (GCS 5). a FLAIR image identifies multiple hyperintense lesions in the splenium of the corpus callosum and in frontal subcortical and periventricular white matter. b GE T2*-weighted image: haemorrhagic shearing injuries are barely visible in the right fronto-opercular and parieto-occipital regions (arrows). c SWI minIP: additional microhaemorrhages are recognisable at the grey matterwhite matter junction of the frontal lobes and in the right parieto-occipital white matter (black arrows). d SWI minIP, reformatted sagittal section shows microhaemorrhages in the corpus callosum (white arrows)
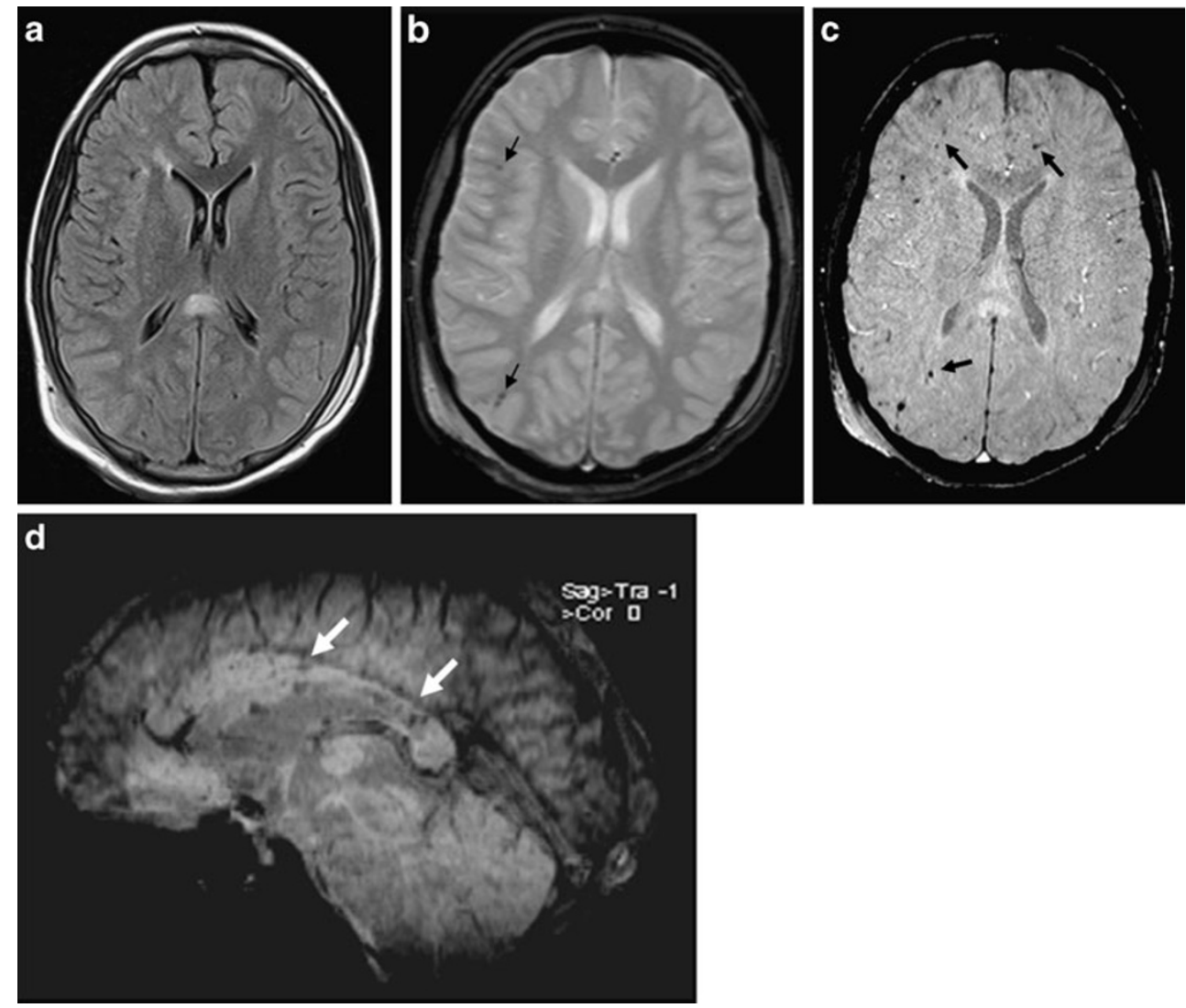
SWI plays a substantial role in the identification and characterisation of cerebral vascular malformations, first of all improving their detection rate. On the other hand, allowing simultaneous visualisation of the different compartments of cerebral AVMs and of the relationship with one another and with the brain parenchyma, it is particularly valuable for therapeutic planning. The clinical usefulness of a similar technique, BOLD MR venography, was first demonstrated by Essig et al. [27].

Phase changes caused by the susceptibility differences between oxygenated and deoxygenated red blood cells and the different relaxation rates between venous and arterial blood provide a natural separation of arteries and veins. The 3D dataset of SWI can therefore be used as an alternative to MR angiography in order to better differentiate afferent arteries from draining veins. This advantage becomes relevant with small AVMs with recent bleeding, in which it is mandatory to identify the nidus in relation to eloquent areas in order to plan the best therapeutic approach, endovascular or surgical or both (Fig. 7). Veins appear dark due to $\mathrm{T} 2 *$ loss and processing of the phase image, while arteries show bright signal from time-of-flight (TOF) inflow enhancement. The use of higher flip angles and shorter TR make it possible to increase the contrast enhancement in the arteries without overly degrading the venography. For the evaluation of high-flow vascular malformations (i.e. AVMs and dural arteriovenous fistulas) the use of both minIP and MIP post-processing techniques can improve the image quality. The identification of small niduses, the exact location of the fistulous point and the depiction of the venous drainage patterns are the most relevant information that should be obtained from this technique. The elevated diagnostic accuracy of SWI compared with digital subtraction angiography (DSA) has been recently reported for the detection of arteriovenous shunting (AVS) in brain AVMs [28].
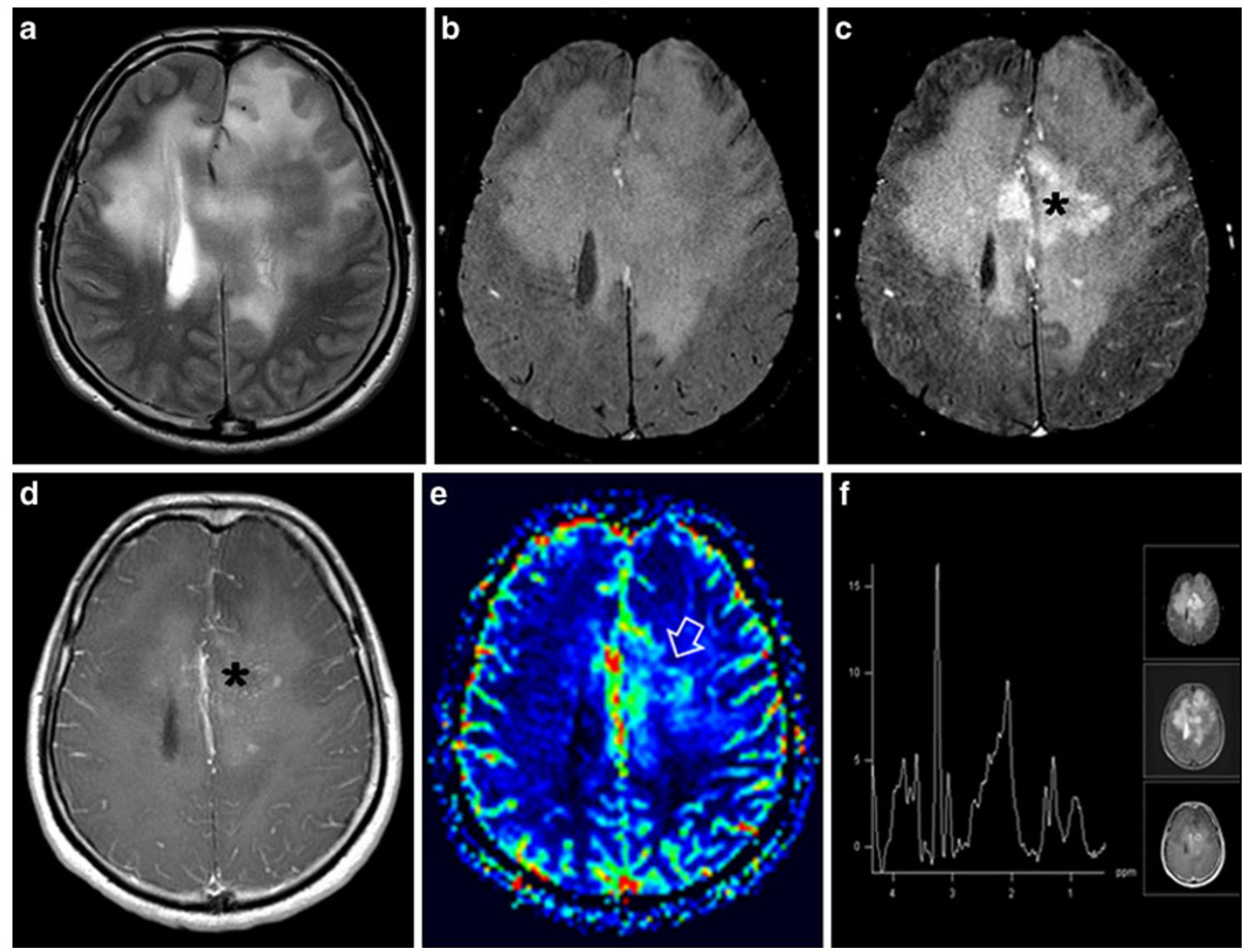

Fig. 14 Characterisation and grading of a glial tumour. A 30-year-old man with dizziness, long-standing behavioural changes and subsequent diagnosis of intra-axial cerebral tumour. a T2-weighted image shows a large frontal infiltrating glioma. b SWI, unprocessed image: absence of intratumoural susceptibility signals (due either to calcifications, haemorrhages or venous vasculature). c Contrast-enhanced SWI: detailed visualisation of the margins of a large anaplastic area (asterisk), which is in good correlation with enhancement character- istics (d), hypervolaemia on PWI relative cerebral blood volume (rCBV) map (e) and MR spectroscopy (f). d Post-contrast T1weighted image. e PWI, rCBV map: area of increased cerebral blood volume (open arrow). f Single-voxel MR spectroscopy, showing inversion of the Cho/NAA ratio and the presence of a lactate peak. Targeted stereotactic biopsy in the supposed necrotic area confirmed the hypothesis of gliomatosis cerebri WHO grade III with several necrotic foci 
Dural AV fistulas (DAVFs) are usually more difficult to identify than AVMs on conventional MRI, especially when venous ectasia is absent, as in type I and II DAVFs; the patients have vague symptoms (i.e. headache or tinnitus) and there are no localising signs [29]. SWI is capable of better identifying the extent of the abnormal venous drainage compared with MR angiography. The conspicuity of the venous structures on SWI can be explained by the combination of a prolonged cerebral circulation time that favours increased oxygen extraction resulting in increased concentration of deoxyhaemoglobin, with the typical venous engorgement (Fig. 8) [30].

Concerning slow-flow vascular malformations (i.e. cavernomas, developmental venous anomalies and capillary telangiectasias) the phase information contained in SWI is responsible for an artefactual enhancement that dramatically improves MRI sensitivity to these pathological conditions. Without SWI, small vascular malformations could be entirely missed by conventional imaging techniques [31].

Individuals with cavernous malformations can present with epilepsy and focal neurological deficits or acute intracranial haemorrhage, although these vascular malformations are often discovered as incidental findings. Cavernomas that have previously bled are usually detectable on routine MRI because of the prominent signal intensity of haemorrhagic products. Cavernomas may also subsequently develop dystrophic calcifications that can be detected on CT. However, these lesions tend to be barely visible when they have not bled, except for faint enhancement after contrast medium administration that is often nonspecific. Patients with multiple lesions can be diagnosed with familial cavernous angiomatosis and should be referred for genetic evaluation and counselling (Fig. 9). Individuals with symptomatic, growing, or haemorrhagic malformations should be considered for surgical resection. Close follow-up after diagnosis and treatment is helpful to identify lesion progression or recurrence [32].

Developmental venous anomalies (DVA) consist of a radially arranged venous complex converging on a centrally located venous trunk, which drains the normal brain parenchyma. They are better displayed with SWI than with T1-weighted contrast-enhanced images (Fig. 10).

Capillary telangiectasias are asymptomatic venous vascular malformations, which are smaller and less common than cavernomas and can sometimes occur in mixed cavernoma/telangiectasia lesions. These may occur sporadically or may be infrequently associated with syndromes, like hereditary haemorrhagic telangiectasia or ataxia telangiectasia. They may also manifest as a result of endothelial injury, such as radiation-induced vascular injury, particularly in children who have received cranial irradiation.
They are characterised by poor contrast enhancement and can therefore be missed on conventional T1-weighted and T2-weighted sequences (Fig. 11).

Ataxia telangiectasia is a rare autosomal recessive disorder characterised by an early-onset of progressive cerebellar ataxia, immunodeficiency, ocular and cutaneous telangiectasia and is associated with an elevated risk of intracranial tumours. SWI can discover brain gliovascular nodules with perivascular haemorrhage and small haemosiderin deposits presumably only in older patients, during the later stages of the disease; however, in our experience this technique can also be a useful screening tool in young patients (Fig. 12) [33].
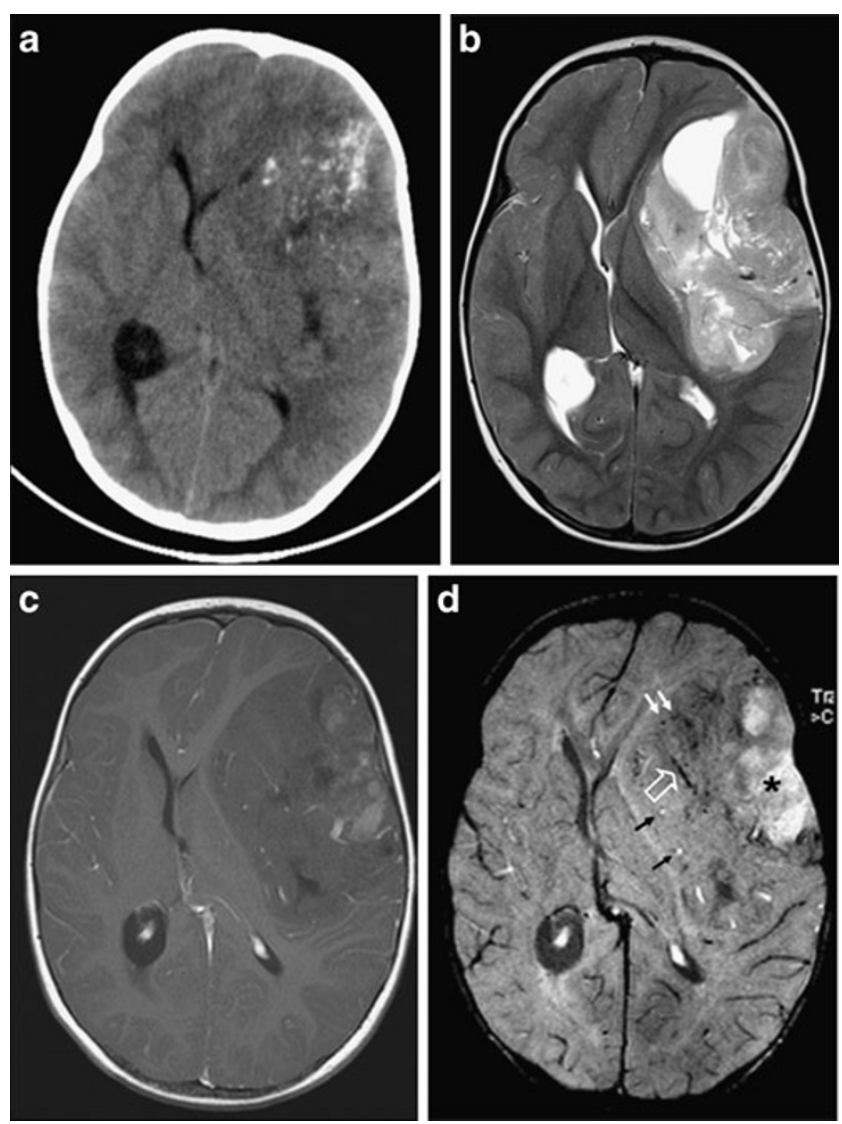

Fig. 15 Internal architecture of a high-grade tumour. A 2-year-old child with headache, vomiting and right hemiparesis. a CT demonstrates a large heterogeneous left frontal tumour with calcified foci. b T2-weighted image: the mass is irregularly hyperintense with cystic changes and small hypointensities representing small vessels and calcifications. c Post-contrast T1-weighted image shows patchy enhancement of the lateral part of the lesion and some vascular structures. d CE-SWI minIP: calcifications appear as punctate hypointensities, unchanged after gadolinium injection (white arrows). Post-processing with minIP algorithm allows the simultaneous visualisation of arteries (hyperintense, black arrows) and veins (linear hypointensities, open arrow) around and inside the tumour. CE-SWI is superior to T1-weighted post-gadolinium sequences in demonstrating a diffuse BBB rupture in the lateral necrotic area (bright signal, asterisk). Histopathology revealed a primary neuroectodermal tumour (PNET) with extensive cystic and necrotic changes and increased vascularity 
Traumatic brain injuries

MRI with GE sequences has been extensively used in the past for investigating young patients with traumatic brain injuries (TBI), either in the acute phase, when the clinical picture of a severe coma is not explained by CT, or months after trauma, in order to understand the causes of an unsuccessful recovery. In both cases, diffuse axonal injuries (DAI) can be responsible for the clinical picture. SWI has been widely used in the identification of smaller haemorrhages due to a shear-strain mechanism of injury, thus refining the prediction of outcome [34]. Haemorrhagic DAI lesions on SWI can be six-times better detected than T2*weighted conventional GE sequences and the recognisable volume of haemorrhage is approximately twofold greater (Fig. 13). Reformatted SWI images in the sagittal plane are particularly helpful in the assessment of corpus callosum haemorrhages. Both number and volume of haemorrhagic lesions correlate with neuropsychological deficits [35].

\section{Intracranial tumours}

Brain MRI predictors of tumour grade include contrast enhancement, oedema, mass effect, cyst formation or necrosis, haemorrhage, metabolic activity and cerebral blood volume. It is well known that the growth of solid tumours, such as gliomas, is dependent on the angiogenesis of pathological vessels. SWI can provide a thorough assessment of the internal angioarchitecture of brain tumours (increased microvascularity inside and beyond the tumour margins), together with the identification of foci of haemorrhage and calcification, thus representing an additional tool in the neuroradiological grading of cerebral neoplasms. The administration of a contrast agent (CESWI) allows discrimination among those three entities, as only blood vessels will change their signal intensity, while calcifications and regions of inactive haemorrhage (which can be differentiated from each other by the evaluation of phase images, as described above) will not. The clinical potential of contrast-enhanced BOLD MR venography at $3 \mathrm{~T}$ and $1.5 \mathrm{~T}$ for the study of brain tumours was first reported by Barth et al. [36], who demonstrated variable venous patterns in various types of tumours and in different parts of the lesions (oedema, contrast-enhancing areas, necrosis), which might represent increased blood supply and particular vascular patterns around fast-growing malignant tumours.

Kim et al. [37] recently assessed the added value provided by SWI in the differential diagnosis of solitary enhancing brain lesions compared with the use of conventional MRI alone. CE-SWI has been found to be equivalent or even superior to CE-T1 images in the evaluation of most tumours with necrotic areas (Figs. 14 and 15): the particular contrast combination within SWI images permits the simultaneous visualisation of the information otherwise obtained by a multimodal imaging approach including CT, CE-T1 SE, FLAIR and T2* conventional GE sequences [38].

Pinker et al. [6] demonstrated a correlation between intratumoural susceptibility effects, positron emission tomography (PET) results and histopathological grading. SWI has been proposed in the evaluation of clinical response to anti-angiogenetic drugs and in the differential diagnosis with pseudo-progression after chemo- and radiotherapy [39]. A correlation with MR PWI has also been attempted [26]. However, larger comparative studies of PWI and SWI are still needed to determine a more precise role of the new techniques in the grading of cerebral neoplasms.

\section{Conclusion}

SWI, which is a combination of GE techniques with phase information, represents a useful tool for the identification and characterisation of vascular malformations and for a better understanding of cerebrovascular diseases. Despite some inherent limitations, SWI has increasing indications for neuroradiology and should be included in the routine imaging protocols of trauma and vascular abnormalities. Further investigation is still needed into its extensive clinical application in neurodegenerative diseases and tumoural pathological conditions.

\section{References}

1. Haacke EM, Mittal S, Wu Z, Neelavalli J, Cheng YC (2009) Susceptibility-weighted imaging: technical aspects and clinical applications, part 1. AJNR Am J Neuroradiol 30:19-30

2. Reichenbach JR, Venkatesan R, Schillinger DJ, Kido DK, Haacke EM (1997) Small vessels in the human brain: MR venography with deoxyhemoglobin as an intrinsic contrast agent. Radiology 204:272-277

3. Reichenbach JR, Essig M, Haacke EM et al (1998) Highresolution venography of the brain using magnetic resonance imaging. Magma 6:62-69

4. Lee BC, Vo KD, Kido DK et al (1999) MR high-resolution blood oxygenation level-dependent venography of occult (low-flow) vascular lesions. AJNR Am J Neuroradiol 20:1239-1242

5. Barnes SR, Haacke EM (2009) Susceptibility-weighted imaging: clinical angiographic applications. Magn Reson Imaging Clin N Am 17:47-61

6. Pinker K, Noebauer-Huhmann IM, Stavrou I et al (2007) Highresolution contrast-enhanced, susceptibility-weighted MR imaging at $3 \mathrm{~T}$ in patients with brain tumors: correlation with positronemission tomography and histopathologic findings. AJNR Am J Neuroradiol 28:1280-1286

7. Nandigam RN, Viswanathan A, Delgado P et al (2009) MR imaging detection of cerebral microbleeds: effect of susceptibility-weighted 
imaging, section thickness, and field strength. AJNR Am J Neuroradiol 30:338-343

8. Vinters HV (1987) Cerebral amyloid angiopathy. A critical review. Stroke 18:311-324

9. Greenberg SM, Finklestein SP, Schaefer PW (1996) Petechial hemorrhages accompanying lobar hemorrhage: detection by gradient-echo MRI. Neurology 46:1751-1754

10. Pettersen JA, Sathiyamoorthy G, Gao FQ et al (2008) Microbleed topography, leukoaraiosis, and cognition in probable Alzheimer disease from the Sunnybrook dementia study. Arch Neurol 65:790-795

11. Fazekas F, Kleinert R, Roob G et al (1999) Histopathologic analysis of foci of signal loss on gradient-echo T2*-weighted MR images in patients with spontaneous intracerebral hemorrhage: evidence of microangiopathy-related microbleeds. AJNR Am J Neuroradiol 20:637-642

12. Smith EE, Gurol ME, Eng JA et al (2004) White matter lesions, cognition, and recurrent hemorrhage in lobar intracerebral hemorrhage. Neurology 63:1606-1612

13. Greenberg SM (2002) Cerebral amyloid angiopathy and vessel dysfunction. Cerebrovasc Dis 13(Suppl 2):42-47

14. Savoiardo M, Erbetta A, Storchi G, Girotti F (2010) Case 159: cerebral amyloid angiopathy-related inflammation. Radiology 256:323-327

15. Staals J, van Oostenbrugge RJ, Knottnerus IL, Rouhl RP, Henskens LH, Lodder J (2009) Brain microbleeds relate to higher ambulatory blood pressure levels in first-ever lacunar stroke patients. Stroke 40:3264-3268

16. Ogg RJ, Langston JW, Haacke EM, Steen RG, Taylor JS (1999) The correlation between phase shifts in gradient-echo MR images and regional brain iron concentration. Magn Reson Imaging 17:1141-1148

17. Good PF, Olanow CW, Perl DP (1992) Neuromelanin-containing neurons of the substantia nigra accumulate iron and aluminum in Parkinson's disease: a LAMMA study. Brain Res 593:343-346

18. Martin WR, Wieler M, Gee M (2008) Midbrain iron content in early Parkinson disease: a potential biomarker of disease status. Neurology 70:1411-1417

19. Vertinsky AT, Coenen VA, Lang DJ et al (2009) Localization of the subthalamic nucleus: optimization with susceptibility-weighted phase MR imaging. AJNR Am J Neuroradiol 30:1717-1724

20. Attems J (2005) Sporadic cerebral amyloid angiopathy: pathology, clinical implications, and possible pathomechanisms. Acta Neuropathol 110:345-359

21. Goos JD, Kester MI, Barkhof F et al (2009) Patients with Alzheimer disease with multiple microbleeds: relation with cerebrospinal fluid biomarkers and cognition. Stroke 40:3455-3460

22. Sveinbjornsdottir S, Sigurdsson S, Aspelund T et al (2008) Cerebral microbleeds in the population based AGES-Reykjavik study: prevalence and location. J Neurol Neurosurg Psychiatry 79:1002-1006

23. Kidwell CS, Saver JL, Villablanca JP et al (2002) Magnetic resonance imaging detection of microbleeds before thrombolysis: an emerging application. Stroke 33:95-98

24. Kidwell CS, Greenberg SM (2009) Red meets white: do microbleeds link hemorrhagic and ischemic cerebrovascular disease? Neurology 73:1614-1615
25. Santhosh K, Kesavadas C, Thomas B, Gupta AK, Thamburaj K, Kapilamoorthy TR (2009) Susceptibility weighted imaging: a new tool in magnetic resonance imaging of stroke. Clin Radiol 64:74 83

26. Mittal S, Wu Z, Neelavalli J, Haacke EM (2009) Susceptibilityweighted imaging: technical aspects and clinical applications, part 2. AJNR Am J Neuroradiol 30:232-252

27. Essig M, Reichenbach JR, Schad LR, Schoenberg SO, Debus J, Kaiser WA (1999) High-resolution MR venography of cerebral arteriovenous malformations. Magn Reson Imaging 17:14171425

28. Jagadeesan BD, Delgado Almandoz JE, Moran CJ, Benzinger TL (2011) Accuracy of susceptibility-weighted imaging for the detection of arteriovenous shunting in vascular malformations of the brain. Stroke 42(1):87-92

29. Cognard C, Gobin YP, Pierot L et al (1995) Cerebral dural arteriovenous fistulas: clinical and angiographic correlation with a revised classification of venous drainage. Radiology 194:671-680

30. Saini J, Thomas B, Bodhey NK, Periakaruppan A, Babulal JM (2009) Susceptibility-weighted imaging in cranial dural arteriovenous fistulas. AJNR Am J Neuroradiol 30:E6

31. de Souza JM, Domingues RC, Cruz LC Jr, Domingues FS, Iasbeck T, Gasparetto EL (2008) Susceptibility-weighted imaging for the evaluation of patients with familial cerebral cavernous malformations: a comparison with T2-weighted fast spin-echo and gradient-echo sequences. AJNR Am J Neuroradiol 29:154-158

32. Batra S, Lin D, Recinos PF, Zhang J, Rigamonti D (2009) Cavernous malformations: natural history, diagnosis and treatment. Nat Rev Neurol 5:659-670

33. Habek M, Brinar VV, Rados M, Zadro I, Zarkovic K (2008) Brain MRI abnormalities in ataxia-telangiectasia. Neurologist 14:192195

34. Mannion RJ, Cross J, Bradley P et al (2007) Mechanism-based MRI classification of traumatic brainstem injury and its relationship to outcome. J Neurotrauma 24:128-135

35. Tong KA, Ashwal S, Holshouser BA et al (2004) Diffuse axonal injury in children: clinical correlation with hemorrhagic lesions. Ann Neurol 56:36-50

36. Barth M, Nobauer-Huhmann IM, Reichenbach JR et al (2003) High-resolution three-dimensional contrast-enhanced blood oxygenation level-dependent magnetic resonance venography of brain tumors at 3 Tesla: first clinical experience and comparison with 1.5 Tesla. Invest Radiol 38:409-414

37. Kim HS, Jahng GH, Ryu CW, Kim SY (2009) Added value and diagnostic performance of intratumoral susceptibility signals in the differential diagnosis of solitary enhancing brain lesions: preliminary study. AJNR Am J Neuroradiol 30:1574-1579

38. Sehgal V, Delproposto Z, Haddar D et al (2006) Susceptibilityweighted imaging to visualize blood products and improve tumor contrast in the study of brain masses. J Magn Reson Imaging 24:41-51

39. Al SA, Buckley R, McHenery C, Pannek K, Coulthard A, Rose S (2010) Distinguishing recurrent primary brain tumor from radiation injury: a preliminary study using a susceptibilityweighted MR imaging-guided apparent diffusion coefficient analysis strategy. AJNR Am J Neuroradiol 31:1049-1054 